\title{
Influence of genetic diversity of seventeen Beauveria bassiana isolates from different hosts on virulence by comparative genomics
}

Zhengkun Zhang ${ }^{\dagger}$, Yang Lu', Wenjing Xu, Li Sui, Qian Du, Yangzhou Wang, Yu Zhao and Qiyun Li*

\begin{abstract}
Background: Beauveria bassiana (B. bassiana) is a famous entomopathogenic fungus that could parasitize on hundreds of insect species, which are being used as an environmentally friendly mycoinsecticide. Nevertheless, the possible effect of genetic diversity of these $B$. bassiana isolates from different hosts on virulence has not been explored before. In order to explore that issue, we compared the genome sequences among seventeen $B$. bassiana isolates from 17 different insects using whole genome re-sequencing, with B. bassiana strain ARSEF 2860 as the reference genome.
\end{abstract}

Results: There were a total of 10,098 missense mutated genes, 720 positively selected genes were identified in 17 strains of B. bassiana. Among these, two genes with high frequency mutations encode the toxin-producing nonribosomal peptide synthase (NRPS) protein. Seven genes undergoing positive selection were enriched in the twocomponent signaling pathway that is known to regulate the fungal toxicity. In addition, the domain changes of three positively selected genes are also directly related to the virulence plasticity. Besides, the functional categorization of mutated genes showed that most of them involved in the biological functions of toxic proteins involved in.

Conclusions: Based on our data, our results indicate that several mutated genes and positively selected genes may underpin virulence of $B$. bassiana towards hosts during infection process, which provide an insight into the potential effects of natural variation on the virulence of B. bassiana, which will be useful in screening out potential virulence factors in B. bassiana.

Keywords: Whole genome re-sequencing, Beauveria bassiana, Genetic diversity, Virulence

\footnotetext{
* Correspondence: qyli@cjaas.com

†Zhengkun Zhang and Yang Lu contributed equally to this work. Jilin Key Laboratory of Agricultural Microbiology, Key Laboratory of Integrated Pest Management on Crops in Northeast China, Ministry of Agriculture, Changchun 130033, Jilin Province, P. R. China
}

(c) The Author(s). 2020 Open Access This article is licensed under a Creative Commons Attribution 4.0 International License, which permits use, sharing, adaptation, distribution and reproduction in any medium or format, as long as you give appropriate credit to the original author(s) and the source, provide a link to the Creative Commons licence, and indicate if changes were made. The images or other third party material in this article are included in the article's Creative Commons licence, unless indicated otherwise in a credit line to the material. If material is not included in the article's Creative Commons licence and your intended use is not permitted by statutory regulation or exceeds the permitted use, you will need to obtain permission directly from the copyright holder. To view a copy of this licence, visit http://creativecommons.org/licenses/by/4.0/ The Creative Commons Public Domain Dedication waiver (http://creativecommons.org/publicdomain/zero/1.0/) applies to the data made available in this article, unless otherwise stated in a credit line to the data. 


\section{Background}

Beauveria bassiana (B. bassiana), a well-known entomopathogenic fungus, is belonged to the family Clavicipitaceae, order Hypocreales [1]. Owing to the high genetic variability, $B$. bassiana exhibits high adaptability to various environmental conditions and thus could parasitize a wide range of insect populations [2]. Currently, $B$. bassiana is actively developed as a biological control tool for various insects in all regions of world [3, 4]. According to $\mathrm{Li}$ et al. report, approximately one million hectares are usually treated with $B$. bassiana to reduce the occurrence of forest insects in China every year [5]. Generally, B. bassiana is thus a generalist with no strict host preference and can be used as an effective and sustainable biological control agent against a myriad of insect pests [6].

Virulence is variable and not universal for a prescribed host and pathogen [7]. Increasing studies have confirmed that the virulence of $B$. bassiana strains from a specific insect host varies frequently [8]. It has been recognized that the strain of $B$. bassiana isolated from a given insect showed low virulence against other insects, and showed stronger virulence against these insects after domestication [9], indicating that the virulence of $B$. bassiana to hosts has a great plasticity. Genome structure and DNA sequence variation in these isolates could be related to many factors in the biology of this fungus. Recently, the re-sequencing of numerous individuals within pathogen species has been as a forceful tool to explore the genetic mechanism in fungal and fungal-host interaction studies [10]. For example, Mburu et al. compared the gene sequences of two $B$. bassiana isolates with different levels of virulence and repellence to the termite, and then some differences were found in the nucleotide sequences of the two isolates of $B$. bassiana, suggesting a genetic basis for the observed intra-specific differences in their virulence against the termite [11]. Valero-Jiménez et al. sequenced the genomes of five isolates of $B$. bassiana with low/high virulence, and finally identified several genes and molecular processes that effect the virulence towards Mosquitoes [12]. These studies provide a better understanding of the natural variation in virulence by genome analysis. Thus, understanding genetic polymorphisms is helpful to understand its potential virulence diversity of $B$. bassiana isolates isolated from different hosts.

In the present study, we aim to gain our knowledge in understanding genomic diversity involved in virulence and host-pathogen interaction for $17 \mathrm{~B}$. bassiana strains by a comparative genomics analysis. Thus, we sequenced the 17 B. bassiana isolates from different hosts compared to the reference genome B. bassiana strain ARSEF 2860. Based on the analysis, understanding the potential factors of genetic variation on the virulence of $B$. bassiana will improve our methods to use this fungus as an effective and sustainable biological control agent against.

\section{Results}

Sequencing and mapping summary

Quality control results of sequencing data of 17 B. bassiana strains were summarized in Table S1. Finally, a total of 160 Gb containing 459 million raw reads were produced, with an average of 27,048,202 reads for each sample. After filtering low quality reads and duplicate reads, 396 million high quality reads were extracted. Approximately $71.22 \%$ of the cleaned reads could be successfully mapped to the $B$. bassiana strain ARSEF 2860 reference genome, with varying 67.30 to $75.30 \%$ among different strains (Table 1). These mapped sequences were used for subsequent analyses.

\section{SNPs and InDels identification}

All clean reads were aligned to the reference genome assembly of B. bassiana strain ARSEF 2860. As a result, a total of 2,779,949 SNPs were identified across $17 \mathrm{~B}$. bassiana isolates genotypes, among which 1,959,716 (70.49\%) are synonymous and 820,233(29.51\%) are nonsynonymous. In addition, a total of 884,811 Indels were obtained, with 423,409 (47.85\%) InDel-Ins and 461,402 (52.15\%) InDel-Del (Table 2). The distributions of SNPtypes and Indel-types of 17 samples were obtained, it showed that majority of SNP variation were belonged to different types including the upstream gene variants $(40.82 \%)$, the synonymous variants $(37.54 \%)$, and the missense variants (15.55\%) (Fig. 1a). Most of the InDels variation were located in the upstream region of the genes (79.15\%) (Fig. 1b). At last, we detected 7421 missense genes are shared by all strains, accounting for $73.49 \%$ of all missense genes $(10,098)$.

\section{Functional annotation}

A hierarchical clustering analysis of the top 50 high frequency missense mutation genes in $17 \mathrm{~B}$. bassiana samples was conducted (Fig. 2a). Clearly, there was no significant numerical difference of each gene in different samples sequenced in this study. However, among different genes, more variation was relatively abundant in four genes (gene_8222, gene_358, gene_6727, and gene_5020) but few variations were relatively lacking in other 46 genes in all $B$. bassiana isolates. Next, we determined the functional categories of these top 50 genes using Gene Ontology database, then their functions were belonged to three general directions. In the biological process category, the top three highly enriched GO terms included binding, catalytic activity, and nucleic acid binding transcription transporter activity. The GO terms of extracellular region, cell part, membrane part, and organelle part were significantly enriched in the cellular component. The molecular function category 
Table 1 Comparison results of sequenced reads of 17 B. bassiana isolates aligned to the reference genome of B. bassiana strain ARSEF 2860

\begin{tabular}{lllllll}
\hline Sample & Clean reads & Mapped-reads & Left mapped reads & Right mapped reads & Pair mapped reads & Map rate \\
\hline S2 & $5,787,346$ & $3,894,494$ & $1,160,251$ & $2,734,243$ & $1,179,149$ & 0.672932636 \\
S3 & $4,753,518$ & $3,307,827$ & $1,063,104$ & $2,244,723$ & $1,078,463$ & 0.695869249 \\
S4 & $6,475,936$ & $4,471,055$ & $1,421,647$ & $3,049,408$ & $1,443,532$ & 0.690410622 \\
S5 & $8,196,298$ & $5,515,918$ & $1,632,128$ & $3,883,790$ & $1,662,691$ & 0.672976751 \\
S6 & $8,562,752$ & $5,769,931$ & $1,720,400$ & $4,049,531$ & $1,749,434$ & 0.673840723 \\
S7 & $12,633,678$ & $9,050,879$ & $3,206,347$ & $5,844,532$ & $3,251,762$ & 0.716408872 \\
S8 & $19,833,546$ & $13,991,144$ & $4,814,073$ & $9,177,071$ & $4,879,648$ & 0.705428268 \\
S9 & $25,549,484$ & $18,221,368$ & $6,473,684$ & $11,747,684$ & $6,551,046$ & 0.713179491 \\
S10 & $15,359,600$ & $11,307,010$ & $4,283,183$ & $7,023,827$ & $4,324,718$ & 0.736152634 \\
S11 & $17,281,180$ & $12,077,193$ & $4,060,203$ & $8,016,990$ & $4,121,883$ & 0.698863909 \\
S13 & $15,289,798$ & $11,493,464$ & $4,526,481$ & $6,966,983$ & $4,559,965$ & 0.751708034 \\
S14 & $20,971,244$ & $15,334,454$ & $5,743,802$ & $9,590,652$ & $5,793,930$ & 0.73121337 \\
S15 & $34,505,488$ & $25,035,584$ & $9,278,608$ & $15,756,976$ & $9,385,046$ & 0.725553686 \\
S18 & $30,045,320$ & $22,624,755$ & $9,075,195$ & $13,549,560$ & $9,134,172$ & 0.753020936 \\
S19 & $63,064,590$ & $45,538,739$ & $17,114,002$ & $28,424,737$ & $17,242,056$ & 0.722096806 \\
S20 & $60,849,634$ & $44,257,288$ & $16,899,228$ & $27,358,060$ & $17,070,101$ & 0.727322173 \\
S21 & $44,537,158$ & $32,079,845$ & $11,709,427$ & $20,370,418$ & $11,832,010$ & 0.72029394 \\
\hline
\end{tabular}

showed a high percentage of metabolic process, cellular process, and single-organism process (Fig. $2 \mathrm{~b}$ ).

\section{Species clustering analysis}

To observe the divergence contained in the individual SNPs among the 17 B. bassiana isolates at the genomic level, we constructed a phylogenetic tree based on the full coding sequence of the genes containing the SNPs obtained from the sequence comparisons. As shown in Fig. 3a, three separate clusters were generated including the isolates of $\mathrm{S} 2 \backslash \mathrm{S} 3 \backslash \mathrm{S} 4 \backslash \mathrm{S} 5 \backslash \mathrm{S} 6 \backslash \mathrm{S} 8 \backslash \mathrm{S} 14$, the isolates of $\mathrm{S} 9 \backslash \mathrm{S} 10 \backslash \mathrm{S} 13 \backslash \mathrm{S} 15 \backslash \mathrm{S} 18 \backslash \mathrm{S} 19 \backslash \mathrm{S} 20 \backslash \mathrm{S} 21$, and the isolates of S7/

Table 2 Number of mutations observed in the seventeen B. bassiana isolates comparing with the reference genome of B. bassiana strain ARSEF 2860

\begin{tabular}{|c|c|c|c|c|c|c|}
\hline Sample & No. synonymous SNPs & No. non-synonymous SNPs & Syn/Nonsyn & SV & InDel-Ins & InDel-Del \\
\hline S2 & 115,930 & 49,487 & 2.342635 & 48 & 10,119 & 11,527 \\
\hline S3 & 114,965 & 48,966 & 2.347854 & 57 & 9,573 & 10,697 \\
\hline S4 & 117,291 & 49,700 & 2.35998 & 27 & 12,343 & 14,010 \\
\hline S5 & 117,039 & 49,616 & 2.358896 & 120 & 13,138 & 14,883 \\
\hline S6 & 116,739 & 49,348 & 2.365628 & 144 & 14,464 & 16,244 \\
\hline S7 & 117,098 & 49,273 & 2.376515 & 132 & 21,341 & 23,608 \\
\hline S8 & 117,232 & 49,111 & 2.387082 & 333 & 27,016 & 29,492 \\
\hline S9 & 115,755 & 48,153 & 2.4039 & 247 & 30,211 & 32,644 \\
\hline $\mathrm{S} 10$ & 116,014 & 48,168 & 2.408528 & 185 & 26,230 & 28,652 \\
\hline S11 & 117,508 & 49,104 & 2.393043 & 179 & 23,756 & 26,421 \\
\hline S13 & 115,278 & 47,742 & 2.414603 & 201 & 27,836 & 30,314 \\
\hline S14 & 116,620 & 48,574 & 2.400873 & 323 & 29,081 & 31,729 \\
\hline S15 & 115,628 & 47,972 & 2.410323 & 333 & 33,174 & 35,704 \\
\hline S18 & 114,229 & 47,079 & 2.426326 & 363 & 34,894 & 37,471 \\
\hline S19 & 107,452 & 44,572 & 2.410751 & 563 & 37,946 & 40,478 \\
\hline $\mathrm{S} 20$ & 111,089 & 46,145 & 2.40739 & 400 & 36,400 & 39,130 \\
\hline S21 & 113,849 & 47,223 & 2.41088 & 508 & 35,887 & 38,398 \\
\hline
\end{tabular}




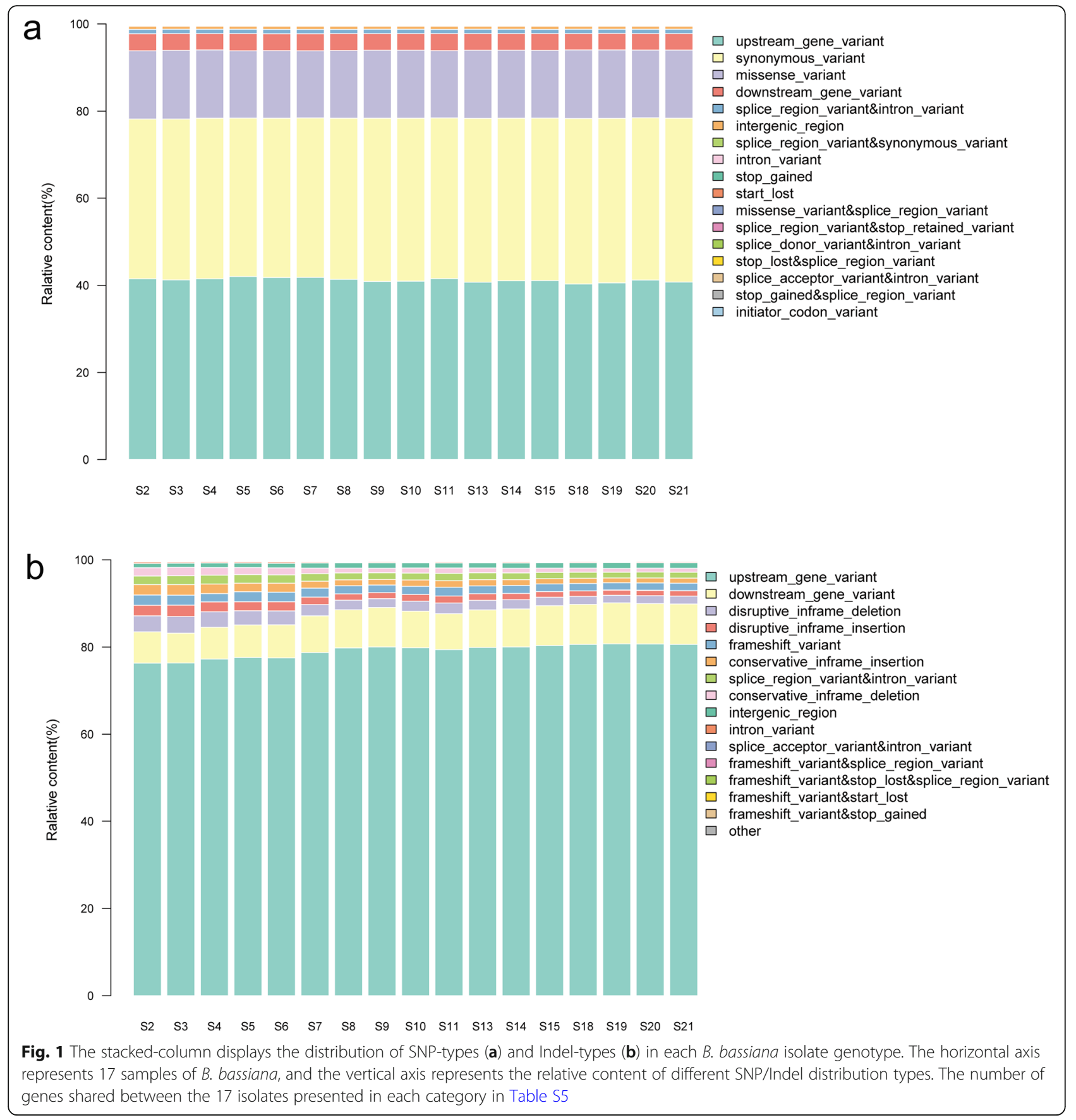

S11. Besides, we performed a principal component analysis (PCA) to examine the genetic relationships among these 17 samples, a clearer separation of three clusters was constructed (Fig. 3b).

\section{$\mathrm{Ka} / \mathrm{Ks}$ analysis}

To confirm the genes subjected to significant selection in of $B$. bassiana domestication, we estimated the statistic $\mathrm{Ka} / \mathrm{Ks}$ with the 10,098 genes contained missense mutation in re-sequencing data of $17 \mathrm{~B}$. bassiana isolates. Finally, a total of 7050 and 720 genes are respectively showed with negative selection or positive section (strong purifying selection) in seventeen samples (Fig. 4a). Subsequently, GO and KEGG pathway analyses were conducted on the positively selected genes. GO enrichment analysis showed highlight a significant enrichment in genes involved in NAD + ADP-ribosyltransferase activity, chromatin, chitin catabolic process, chromatin assembly or disassembly, phosphoenolpyruvate-dependent sugar phosphotransferase system, tRNA-intron endonuclease complex, actin 

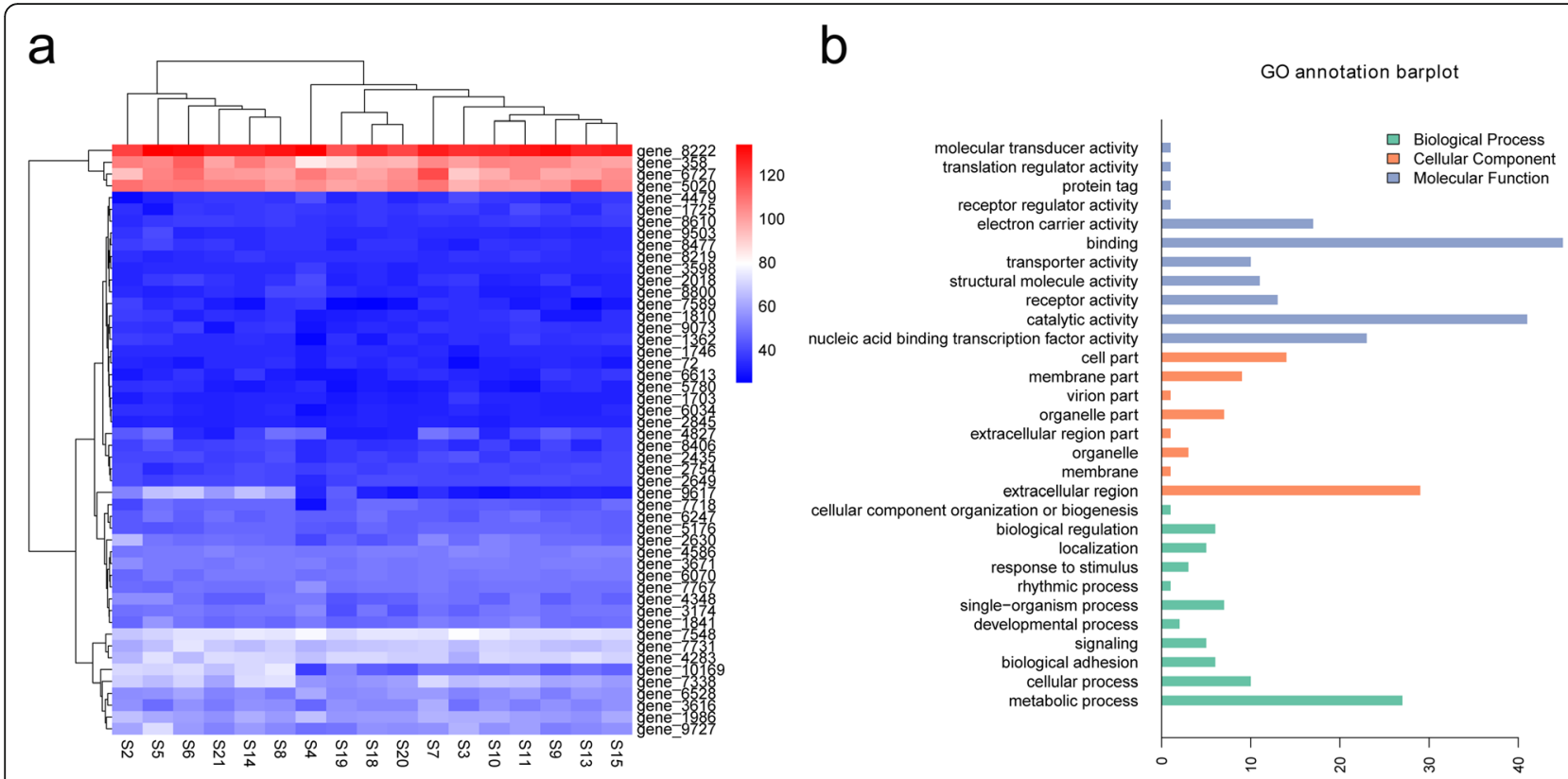

Fig. 2 The mutation numbers and annotation information of 50 genes with high frequency of missense mutations. a A hierarchical clustering analysis showing the relative difference in the number of SNPs in these top 50 genes in 17 B. bassiana samples. The red 4 genes exhibit a higher number of SNP mutations in 17 B. bassiana samples, while the blue 46 genes exhibit a low number of SNP mutations in 17 B. bassiana samples. b Gene Ontology (GO) functional annotation of the top 50 genes with missense mutations in the re-sequencing data of 17 B. bassiana isolates. The results are summarized in three main categories: biological process (BP), cellular component (CC) and molecular function (MF)

a

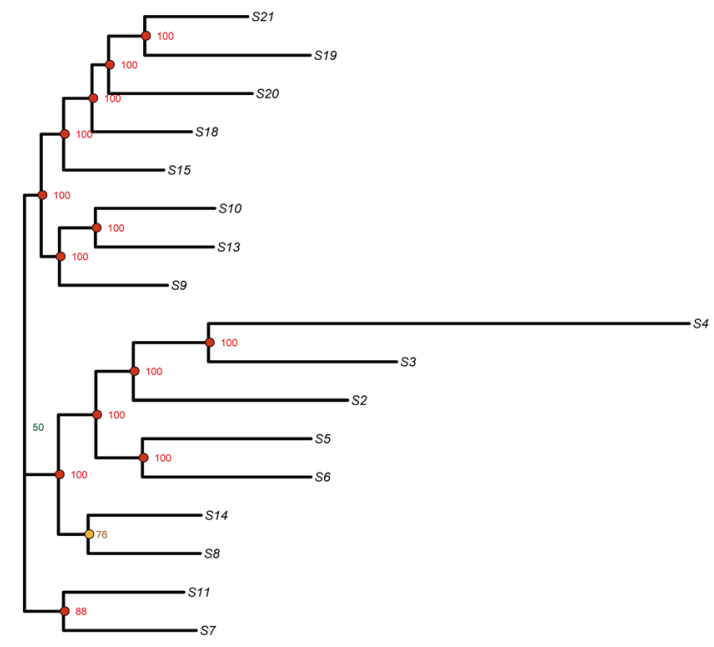

b

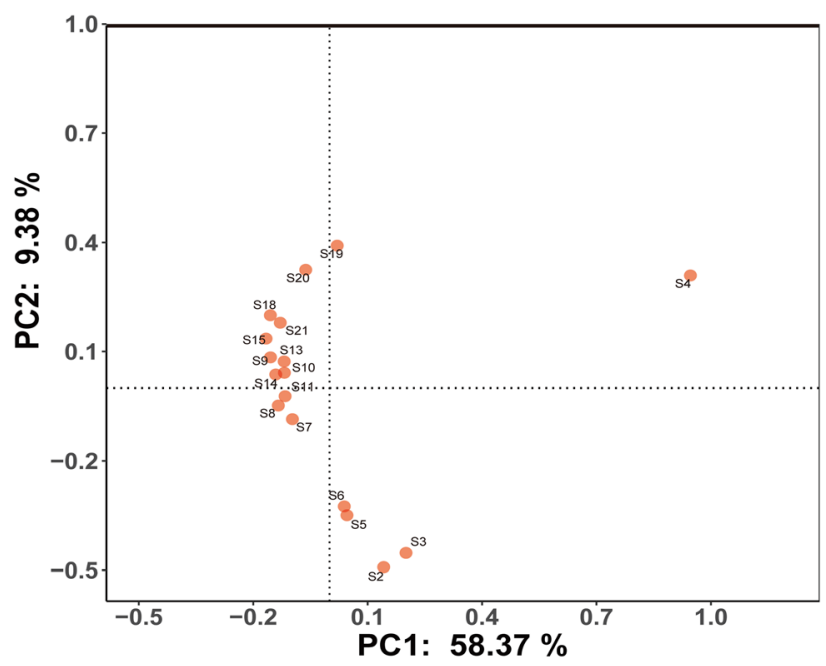

Fig. 3 The genetic diversity of the missense SNPs in the re-sequencing data of seventeen B. bassiana isolates. a A phylogenetic tree was constructed. All missense mutated nucleotide sequences of 17 strains of $B$. bassiana were used to construct an evolutionary tree based on the similarity of SNP missense mutations according to modelfinder and iqtree software. The phylogenetic tree was constructed with 100 bootstrapping replicates. Similar missense mutations were distributed in the same cluster. b Principal component analysis (PCA) analysis of 17 samples based on missense SNPs. Principle component plot constructed by Plink software 

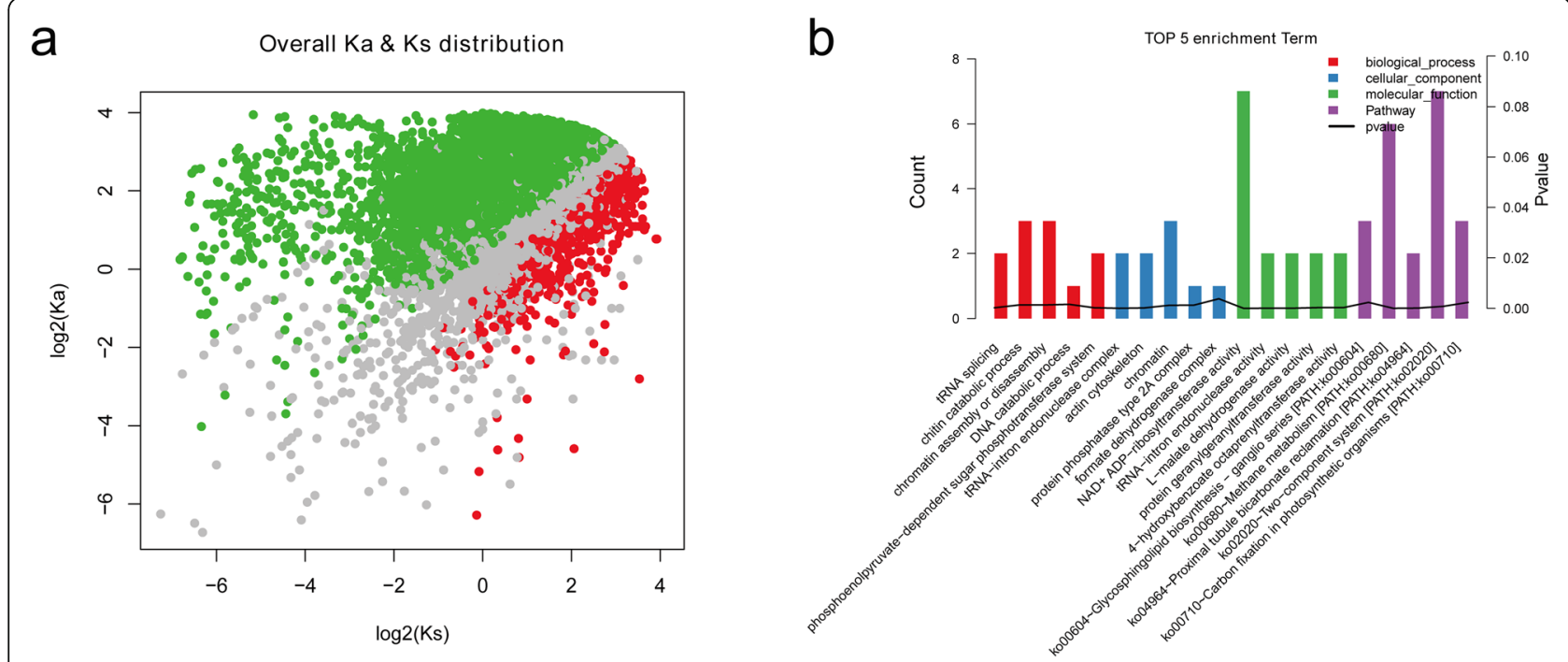

Fig. $4 \mathrm{Ka}$ (non-synonymous)/Ks (synonymous) analysis and functional annotation of the genes with missense mutations in the re-sequencing data of $17 \mathrm{~B}$. bassiana isolates. a The overall Ka/Ks distribution. The red dots represent genes with positive selection in 17 samples, while the green dots represent genes with negative selection in 17 samples. b Gene Ontology (GO) and Kyoto Encyclopedia of Genes and Genomes (KEGG) functional enrichment analysis of 720 positive selection genes. The $\mathrm{GO}$ annotation results were summarized in three main categories: biological process (BP), cellular component (CC) and molecular function (MF). The KEGG annotation results were summarized in pathways

cytoskeleton, tRNA-introendonuclease activity, L-malate dehydrogenase activity, protein geranygeranyltransferase activity, 4-hydroxybenzoate octaprenyltransferase activity, and so on (Fig. 4b). The KEGG pathway analysis revealed that the genes were overrepresented in 5 pathways, of which the highest represented pathway is glycosphingolipid biosynthesis-ganglio series (PATH: ko00604), followed by methane metabolism (PATH: KO00680), proximal tubule bicarbonate reclamation (PATH: KO04964), two component systems (TCSs) (PATH: KO02020), and carbon fixation in photosynthetic organisms (PATH: ko00710) (Fig. 4b). Herein, a total of seven genes (gene_ 664, gene_2709, gene_3056, gene_3362, gene_7254, gene 7433, gene_8336) with positive selection were abundantly enriched in this pathway, and the proteins encoded by these genes were Oxidoreductase short-chain dehydrogenase/reductase family, Galactoside O-acetyltransferase, Ulp1 protease family protein, UcrQ family protein, AcylCoA $\mathrm{N}$-acyltransferase, Ubiquinol-cytochrome c reductase complex subunit, and CAAX amino terminal protease (Table 3). The corresponding mutation information of these seven genes was presented in Table S2.

\section{Structural variations detection}

The detection of SVs was achieved by making use of BreakDancer programs. A total of 4163 individual SVs were predicted, with an average of $245 \mathrm{SVs}$ in each $B$. bassiana strain genotype (Table 2). Most of the SVs were unique in each sample, and only 6 structural variation loci were shared among different samples, as shown in Table S3.

\section{Protein domain analysis}

Finally, we investigated the effect of the missense mutations of the 720 positively selected genes on protein structure by the comparation of 17 samples. Collectively, 13 putative domains encoded by 13 protein-coding genes were identified, of which the genome regions encoding

Table 3 The information of the seven positively selected genes that are enriched in the two component systems

\begin{tabular}{|c|c|c|c|c|}
\hline Gene & Protein-description & Protein & EC & $\mathrm{KO}$ \\
\hline gene_664 & Oxidoreductase short-chain dehydrogenase/reductase family & XP_008593983.1 & 1.1.1.- & K18347 \\
\hline gene_2709 & Galactoside O-acetyltransferase & XP_008596028.1 & 2.3.1.- & K15853 \\
\hline gene_3056 & Ulp1 protease family protein & XP_008596375.1 & 3.4.22.- & K12292 \\
\hline gene_3362 & UcrQ family protein & XP_008596681.1 & 1.10.2.2 & K00411 \\
\hline gene_7254 & Acyl-CoA N-acyltransferase & XP_008600573.1 & 2.3.1.- & K15853 \\
\hline gene_7433 & Ubiquinol-cytochrome c reductase complex subunit & XP_008600752.1 & 1.10.2.2 & K00411 \\
\hline gene_8336 & CAAX amino terminal protease & XP_008601655.1 & 3.4.22.- & K12292 \\
\hline
\end{tabular}


proteins with MutS_IV domain, Zn_clus domain, Fungal_trans_2 family, Metallophos domain, and Metallophos_2 domain were most richest and shared by seventeen samples (Table 4).

\section{Discussion}

B. bassiana, originated from the different hosts shows distinct virulence and pathogenic. Identification of the genetic variation of $B$. bassiana strains infecting the different hosts would improve our understanding of the differences towards the hosts, and may help clarify the potential virulence of $B$. bassiana against the insects. Recently, several researches have been performed to investigate the genetic variation within the pathogen originated from different hosts using re-sequencing technology and bioinformatics [13-15]. Based on that, it is of great significance to investigate the genomic alterations of $17 \mathrm{~B}$. bassiana isolates of the fungus with different levels of virulence to other Fungi in the present study.

Firstly, 17 strains of $B$. bassiana originated from different hosts were sequenced by genome re-sequencing. In general, the alignment rate obtained by comparing the assembled sequences is not comparable, and the mutation frequency cannot be known. For example, if a mutation occurs at a partial site of a sample, i.e. a heterozygote appears, the mutation cannot be counted. Therefore, we chose to match the quality-controlled clean reads to the reference genome of $B$. bassiana strain ARSEF 2860 [16], which identify the difference loci and calculate their genotype frequency, so as to be more accurate. The results showed approximately $71.22 \%$ of the cleaned reads were mapped to the $B$. bassiana strain ARSEF 2860, and a total of 2,779,949 SNPs, 884,811 Indels, and 4163 SVs, were identified in seventeen genomes when compared to the reference

Table 4 Genes and domain prediction information for domain changes in the seventeen B. bassiana isolates

\begin{tabular}{|c|c|c|c|c|c|}
\hline Gene ID & $\mathrm{Hmm}_{-}$accession & Hmm_name & Type & Compare with reference & Samples \\
\hline gene_10,314 & PF05190.15 & MutS_IV & Domain & lost & $\begin{array}{l}\text { S10; S11; S13; S14; S15; S18; S19; S2; S20; S21; } \\
\text { S3; S4; S5; S6; S7; S8; S9 }\end{array}$ \\
\hline gene_3355 & PF13637.3 & Ank_4 & Domain & lost & S4 \\
\hline gene_3355 & PF13857.3 & Ank_5 & Domain & add & S4 \\
\hline gene_3930 & PF00172.15 & Zn_clus & Domain & add & $\begin{array}{l}\text { S10; S11; S13; S14; S15; S18; S19; S2; S20; S21; } \\
\text { S5; S6; S7; S8; S9 }\end{array}$ \\
\hline gene_3930 & PF00172.15 & Zn_clus & Domain & add & S3 \\
\hline gene_3930 & PF00172.15 & Zn_clus & Domain & add & S4 \\
\hline gene_3930 & PF11951.5 & Fungal_trans_2 & Family & add & $\begin{array}{l}\text { S10; S11; S13; S14; S15; S18; S19; S2; S20; S21; } \\
\text { S3; S5; S6; S7; S8; S9 }\end{array}$ \\
\hline gene_3930 & PF11951.5 & Fungal_trans_2 & Family & add & S4 \\
\hline gene_3959 & PF00023.27 & Ank & Repeat & lost & $\mathrm{S} 11 ; \mathrm{S} 5 ; \mathrm{S} 6$ \\
\hline gene_4478 & PF00023.27 & Ank & Repeat & lost & S11; S15; S19; S4; S9 \\
\hline gene_4478 & PF12796.4 & Ank_2 & Repeat & lost & S10; S20; S21; S3; S6 \\
\hline gene_5131 & PF00023.27 & Ank & Repeat & lost & S14; S15; S18; S8; S9 \\
\hline gene_5131 & PF13857.3 & Ank_5 & Domain & add & S10; S11; S14; S15; S2; S3; S5; S8; S9 \\
\hline gene_5131 & PF13857.3 & Ank_5 & Domain & add & S18; S20 \\
\hline gene_5369 & PF00149.25 & Metallophos & Domain & lost & $\begin{array}{l}\text { S10; S11; S13; S14; S15; S18; S19; S2; S20; S21; } \\
\text { S3; S4; S5; S6; S7; S8; S9 }\end{array}$ \\
\hline gene_5369 & PF12850.4 & Metallophos_2 & Domain & add & $\begin{array}{l}\text { S10; S11; S13; S14; S15; S18; S19; S2; S20; S21; } \\
\text { S4; S5; S7; S8; S9 }\end{array}$ \\
\hline gene_5369 & PF12850.4 & Metallophos_2 & Domain & add & S3; S6 \\
\hline gene_5651 & PF01636.20 & $\mathrm{APH}$ & Family & add & $\mathrm{S} 13 ; \mathrm{S} 18$ \\
\hline gene_7731 & PF00023.27 & Ank & Repeat & add & S4 \\
\hline gene_9165 & PF13414.3 & TPR_11 & Repeat & add & $\begin{array}{l}\text { S10; S11; S13; S14; S15; S18; S19; S2; S20; S21; } \\
\text { S4; S6; S7; S8; S9 }\end{array}$ \\
\hline gene_9165 & PF13414.3 & TPR_11 & Repeat & add & S3 \\
\hline gene_9207 & PF13176.3 & TPR_7 & Repeat & add & S18 \\
\hline gene_9900 & PF00023.27 & Ank & Repeat & lost & S10; S18; S20; S21; S4; S9 \\
\hline gene_9932 & PF13516.3 & LRR_6 & Repeat & add & $\mathrm{S} 11 ; \mathrm{S} 21 ; \mathrm{S} 3$ \\
\hline
\end{tabular}


genome. As the most credible genome-wide genetic markers, single-nucleotide polymorphisms (SNPs) have been widely applied to characterize potentially adaptive genetic variation [17]. Herein, the proportion of nonsynonymous SNPs among all the SNPs is $29.51 \%$, which is supported by Valero-Jiménez's work of 87,119 SNPs between Bb5078 and Bb8028, and 467,292 SNPs between $\mathrm{Bb} 4305$ and Bb8028 [18], revealing a high level of genetic diversity among different strains of $B$. bassiana. Among the Indels, the number of InDel-Ins variants $(423,409,47.85 \%)$ is almost equal to the InDel-Del variants $(461,402,52.15 \%)$, and most of the variants were located in the upstream region of the genes (79.15\%). Furthermore, structural variation is known to be a key component of phenotypic diversity in organisms [19]. In this study, all types of the identified SVs were interchromosomal translocations (CTXs), which may be due to the incomplete splicing of genomes caused by the existence of a considerable amount of gaps in the fungus genome [20].

Secondly, a hierarchical clustering analysis was carried out on the top 50 genes with missense SNPs in 17 samples. The results showed that there was no significant difference in the frequency of missense SNP mutations among different hosts, which revealing the population identity of the seventeen sequenced $B$. bassiana isolates. However, significant nucleotide variations were identified in four genes (gene_8222, gene_358, gene_6727, and gene_5020) in all samples, and two of them encoding the common protein non-ribosomal peptide synthase (NRPS, gene_8222 and gene_6727). NRPS is a key mechanism for the biosynthesis of bioactive metabolites in fungi and plays an important role in the production of fungaltoxin. For example, NRP synthetase Pes3 has been found to contribute to Aspergillus. fumigatus virulence, and disruption of pes 3 resulted in an augmented virulence phenotype in invertebrate for invasive aspergillosis. NRP synthetase mgoA has a role in the virulence of Pseudomonas. syringae pv. Syringae, and A Tn5 mutant disrupted in mgoA was defective in mangotoxin production and virulence. NRP synthetase gliP was predicted to be involved in gliotoxin production, and mutants with a disrupted gliP locus failed to produce gliotoxin [21-23]. Based on these findings, nonribosomal peptide synthesis (NRPS) is considered to be a documented virulence factor for fungi, its mutations affect the different virulence of the hosts. Importantly, the two genes encoding NRPS in this study showed more missense mutations in $17 \mathrm{~B}$. bassiana trains, which may be closely related to the maintenance of the pathogenesis during the evolution process, but the specific virulence effects of each mutation need to be further explored. Furthermore, GO annotations showed that the functions of mutated genes were mainly related to binding, catalytic activity, extracellular region, metabolic process, and nucleic acid binding transcription transporter activity. Mutated genes involved in binding, transport and other biological processes may be associated with increased interaction between virulence associated proteins and hosts when $B$. bassiana exerts a toxic infection. Additionally, among these groups, the variation involved in the metabolic process may be related to the production of antibacterial compounds, it is very important for $B$. bassiana to resist microbial competition in the process of killing insects [24].

Thirdly, the phylogenetic relationships and principal component analysis (PCA) between isolates of B. bassiana were determined using the whole missense SNPs. Three distinct $B$. bassiana clusters were formed, namely the isolates of S2 $\backslash \mathrm{S} 3 \backslash \mathrm{S} 4 \backslash \mathrm{S} 5 \backslash \mathrm{S} 6 \backslash \mathrm{S} 8 \backslash \mathrm{S} 14 \backslash \mathrm{S} 19$, the isolates of $\mathrm{S} 2 \backslash \mathrm{S} 9 \backslash \mathrm{S} 10 \backslash \mathrm{S} 13 \backslash \mathrm{S} 15 \backslash \mathrm{S} 18 \backslash \mathrm{S} 19 \backslash \mathrm{S} 20 \backslash \mathrm{S} 21$, and the isolates of S7/S11. Previously, the study of Ghikas et al. revealed that seven phylogenetic clusters in B. bassiana that consisted of isolates which had common climate features but did not exhibit any geographic distribution [25]. Our finding is partly in agree with the earlier suggestions that B. bassiana is not monophyletic, but rather is composed of several clusters that are morphologically identical [25-27], which jointly confirmed a high genetic diversity of $B$. bassiana. Additionally, the results showed that similar mutations occurred in B. bassiana strains in each cluster during evolution, which indicated a close relationship among $B$. bassiana isolates isolated different hosts in each cluster, but the relationship between the virulence of $B$. bassiana isolates in each cluster is not clear and further studies are needed. Furthermore, in the PCA plot, an obvious separation of three clusters was found, which also revealed a clear genetic differentiation among these 17 samples.

In previous study, genes with a signature of positive selection is possible to result in the adaptation to a dynamic environment and may be associated with fungi virulence [18]. Herein, we calculated the $\mathrm{Ka} / \mathrm{Ks}$ values to identify the genes subjected to selection in the evolution of $B$. bassiana. Finally, a total of 7050 genes with $\mathrm{Ka} /$ $\mathrm{Ks}>1$ and 720 genes with $\mathrm{Ka} / \mathrm{Ks}<1$ are confirmed, respectively. Among genes predicted to be under strong purification selection, there is enrichment for GO categories involving NAD + ADP-ribosyltransferase activity, chromatin, chromatin assembly or disassembly, chitin catabolic process, and actin cytoskeleton. In Salmonella, $s p v$ genes can establish successful systemic infection in experimental animals, and SpvB virulence-associated protein has recently been shown to contain the ADPribosyltransferase domain, suggesting that NAD + ADPribosyltransferase activity is associated with the regulation of virulence [28]. Chromatin is densely packed with the nucleosome that contains the genetic information in 
eukaryotes. Many events involve chromatin alterations that are crucial for virulence and have been considered to be a hopeful antifungal targets. For example, chromatin function affects pathogenicity of the most prevalent human fungal pathogen Candida albicans [29]. Earlier study has confirmed that the important role of drastic actin cytoskeleton reorganization in various different aspects of host-pathogen interaction [30]. Chitin is one of the main components of insect cuticle that acts as the first barrier against fungal pathogens. The entomopathogenic fungi $B$. bassiana could secrete several chitindegrading enzymes, such as Endo chitosanase and Chitinase D [31], which are the critical cuticle-degrading enzymes and act synergistically with proteases to digest insect cuticle, and thus cause the enhancement of $B$. bassiana virulence [32]. Additionally, KEGG pathways analysis suggested the positively selected genes were highly enriched in glycosphingolipid biosynthesis-ganglio series, methane metabolism, proximal tubule bicarbonate reclamation, two component systems, and carbon fixation in photosynthetic organisms with some important physiological functions. Of which, two component systems (TCSs) are widely distributed in various bacteria and play important roles in the organisms in the face of different external stimuli via controlling gene expression [33]. In the present study, seven genes among these 17 $B$. bassiana strains with positive selection were abundantly enriched in this pathway. Increasing studies have revealed the involvement of this pathway in regulating resistance processes and diverse virulence in a wide range of pathogenic bacteria, such as Mycobacterium tuberculosis [33], Salmonella typhimurium [34], Staphylococcus aureus [35], Pseudomonas aeruginosa [36]. Taken together, GO and KEGG annotation analysis identified some positive selection genes associated with these above functional processes in the present study, which may serve as potential factors for virulence mechanisms, providing new support for studying of the virulence of $B$. bassiana strains.

Finally, a comprehensive protein domain analysis was performed on 720 positively selected genes with missense mutations. Significant differences in 13 domains encoded by 13 protein-coding genes were identified. Among these, three domain proteins involved in the host-pathogen interaction, such as ankyrin repeat domain protein, Metallophos domain protein and MutS domain $\mathrm{V}$ that were encoded by gene-7731, gene-5369, and gene-10,314 may affect host-pathogen interactions. For example, Zahrt et al. found that the alteration in virulence on Salmonella was the direct result of the inactivation of both of the mutS and recD gene products, however, S. typhimurium carrying mutations in either one of these genes behaved similarly to the wild-type strain and without attenuating virulence [37]. Ankyrin repeats are essential to regulate protein-protein interactions involved in a variety of host processes, including cytoskeletal motility, tumor suppression, and transcriptional regulation [38, 39]. Besides, Lamb et al. reported that myxoma virus as an effective vertebrate pest control agent, ANK-R mutant can both activate NF$\kappa \mathrm{B}$ pathway and engender virus-induced apoptosis [40]. The Metallophos domain is located in the conserved region of the Ser/Thr protein phosphatase gene, which is the central mediator of phosphorylation-dependent signals in various pathogenic bacteria and eukaryotes and is responsible for achieving metabolic control [41]. Earlier research has unveiled the important role of Ser/Thr phosphorylation in the virulence of Mycobacterium tuberculosis, which could regulate many intracellular metabolic processes and interfere with signaling pathways of the infected host cell [42]. In summary, based on the correlation between the virulence and these domains from different hosts, the altered protein domain in these $17 \mathrm{~B}$. bassiana strains may be closely related to its plasticity of virulence. Besides, other genes deletion or genes acquisition may also be critical in virulence evolution. Of course, further study is needed in the future.

\section{Conclusion}

According to the plasticity and variability of fungal virulence, we sequenced and compared the genome of seventeen $B$. bassiana isolates obtained from different insect hosts. Subsequently, the relationship between stable genetic variation and virulence function shared by 17 strains of B. bassiana in different hosts, and the correlation between mutated genes and virulence effect of $B$. bassiana were analyzed. A number of genes with stable mutations in these $B$. bassiana isolates have been established. Among them, some key factors including NRPS mutations, domain changes of ankyrin repeat domain protein, Metallophos domain protein, and MutS domain occurred in B. bassiana isolates may be crucial for the virulence of B. bassiana. Besides, the 720 positive selection genes and the top 50 genes with missense mutations were significantly enriched in some important biological processes, and pathways may also be related to the virulence of $B$. bassiana against hosts. The study provided the relevant theoretical basis for targeted changes in the virulence effect of $B$. bassiana. In this case, we think the virulence of $B$. bassiana might be influenced by natural variation in the process of evolution, which may benefit future molecular investigations of insect-fungus interactions and promote the development of $B$. bassiana as cost-effective and sustainable mycoinsecticides. However, do these genetic mutations really play a role in virulence or how do it regulate virulence in biological processes, which is interested and will need to further study. 


\section{Methods}

\section{Fungal strains and culture conditions}

This study employed seventeen isolates of B. bassiana that were obtained from 17 different insects (Table S4). All isolates were preserved on Sabouraud dextrose agar (SDAY: $4 \%$ glucose, $1 \%$ peptone, $1.5 \%$ agar and $1 \%$ yeast extract) and maintained at $25^{\circ} \mathrm{C}$.

\section{Genome sequencing and mapping}

Genomic DNA of 17 isolates of B. bassiana was extracted using the $\mathrm{CTAB}$ method as the previous report [43]. DNA concentration and purity were checked on NanoDrop (Thermo Fisher Scientific Inc. Waltham, MA, USA), and the qualified DNAs were used for library construction. Sequencing libraries for each individual were established using the standard Illumina protocol (Illumina, San Diego, CA, USA) and sequenced using HiSeq 2000 instruments (Illumina inc., San Diego, CA, USA) according to the manufacturer's protocol by Sangon Biotech (Shanghai) Co., Ltd., Shanghai, China. The generated raw reads were processed using the FASTX-Toolkit version 0.0.13 (http://hannonlab.cshl.edu/fastx_toolkit) to filter the low-quality reads $(<10)$, reads shorter than $50 \mathrm{nt}$ and duplicated reads [44]. After the filtering, the high quality reads were extracted and mapped to the reference genome of $B$. bassiana strain ARSEF 2860 [16]. The alignment processing was conducted using BWA (version V0.7.12-r1039) with default parameters [45].

\section{SNP and Indel detection}

Picard tools version 1.92 (http://picard.sourceforge. net) was used to discard duplicates, and the obtained BAM files were further processed using SAMtools 1.4.1 (samtools.sourceforge.net) [46]. SNPs and indel calling was performed with the GATK v2.4-9 (software broadinstitute.org/gatk/) UnifiedGenotyper [47]. After that, an initial GATK VariantFiltration step was performed with the filtering conditions: For SNPs: with the filter expression 'QD $<2.0 \|$ FS $>60.0$ || MQ < 40.0 || HaplotypeScore > 13.0 || MappingQualityRankSum < -12.5 || ReadPosRankSum < -8.0'; for Indel: QD < 2.0 || FS > 200.0 || ReadPosRankSum < 20.0. The annotations for genes with SNPs and Indels were completed using the software SNPEFF (version $4.3 \mathrm{k}$ ) variant effect prediction (http://snpeff.sourceforge.net/) [48].

\section{Species clustering analysis}

To determine the evolutionary relationship among $B$. bassiana originates from different hosts. The optimal nucleic acid replacement mode was found by modelfinder. Then a phylogenetic tree was conducted using iqtree with the bootstrapping of 100 replicates [49], and further presented using an R package, ggtree. PCA was carried out using the software Plink1.9 [50] on the missense SNPs that for all genotyped individuals, and presented using ggplot2.

\section{$\mathrm{Ka} / \mathrm{Ks}$ analysis}

The positively selected genes among the genomes of 17 $B$. bassiana isolates were searched using the bidirectional best hit $(\mathrm{BBH})$ method [51]. The ratio of nonsynonymous SNP (Ka) to synonymous SNP (Ks) in each gene was defined, including neutral $(\mathrm{Ka} / \mathrm{Ks}=1)$, negative $(\mathrm{Ka} / \mathrm{Ks}>1)$ and positive $(\mathrm{Ka} / \mathrm{Ks}<1)$ selection. The Ka and $\mathrm{Ks}$ in each missense mutant genes were calculated, and the ratio of $\mathrm{Ka}$ to $\mathrm{Ks}$ was estimated by the Codeml model of the program of phylogenetic analysis by maximum likelihood (PAML). To confirm functionally coherent gene-sets that showed $\mathrm{Ka} / \mathrm{Ks}$ values significantly $<1$, Gene Ontology (GO) enrichment analysis and Kyoto Encyclopedia of Genes and Genomes (KEGG) analysis were performed.

\section{Functional annotation}

A hierarchical clustering analysis of the top 50 genes with missense SNPs was conducted by the clustergram function in the Matlab Bioinformatics toolbox with minor changes. GO analysis was performed with Fisher's Exact Test and classified under three categories: biological process (BP), cellular component (CC), and molecular function (MF) [52]. Meanwhile, the detected positively selected genes were mapped to the terms of KEGG (http://www.genome.jp/kegg/) database to explore main biological functions [53], and the assignment of differential genes to different gene families was realized using Pfam (protein families) (http://pfam.xfam.org/ ) annotation [54].

\section{Structure variations (SVs) detection}

Structural variants was determined using BreakDancer (v1.1.2) software [55], containing large fragments of SV types, including deletions (DEL), insertions (INS), inversions (INV), inter-chromosomal translocations (CTX), and so on.

\section{Protein domains analysis}

To explore the effect of nonsynonymous SNPs in the positively selected genes on protein domains, we first identify the mutant amino acid sequences using Bioperl. Then, the hidden Markov model (HMM) profile for the NBS domain was applied to search the protein sequences in the B. bassiana database using hmmsearch in HMMER (v3.1b) with e-value <0.00001 [56]. 


\section{Supplementary information}

Supplementary information accompanies this paper at https://doi.org/10. 1186/s12864-020-06791-9.

Additional file 1: Table S1. Quality control results of sequencing data of 17 B. bassiana strains. Table S2. Mutation information of the seven positively selected genes that are enriched in the two component systems. Table S3. The formation of the structure variants shared by seventeen B. bassiana isolates genomic sequences. Table S4. The information of the hosts of seventeen B. bassiana isolates. Table S5. The number of genes shared between the 17 isolates presented in each category in Fig. 1.

\section{Abbreviations}

B. bassiana: Beauveria bassiana; PCA: Principal component analysis; SNPs: Single-nucleotide polymorphisms; CTXs: Inter-chromosomal translocations; NRPS: Nonribosomal peptide synthesis; TCSs: Two component systems; BBH: Bidirectional best hit; GO: Gene Ontology; KEGG: Kyoto Encyclopedia of Genes and Genomes; BP: Biological process; CC: Cellular component; MF: Molecular function; SVs: Structure variations; DEL: Deletions; INS: Insertions; INV: Inversions; HMM: Hidden Markov mode

\section{Acknowledgements}

Not applicable.

\section{Authors' contributions}

Conceptualization: QYL; Data curation: ZKZ, YL; Formal analysis: WJX, LS; Funding acquisition: QYL; Investigation: YZW; Methodology: ZKZ, YL; Project administration: QYL; Resources: QD, YZW, YZ; Software: WJX, QD; Supervision: QYL; Validation: QYL; Visualization: LS, YZ; Roles/Writing - original draft: ZKZ, YL; Writing - review \& editing: QYL. All authors have read and approved the manuscript.

\section{Funding}

This research was funded by grants from National Key R\&D Programs (Grant No.: 2017YFD200608) and Jilin Provincial Agricultural Science and Technology Innovation Project (Grant No.: CXGC 2017JQ016). The funders had no role in study design, data collection and analysis, decision to publish, or preparation of the manuscript.

\section{Availability of data and materials}

The datasets generated and analysed during the current study are available in the SRA database of National Center for Biotechnology Information [https:// www.ncbi.nlm.nih.gov/sra/PRJNA626696 and SRA accession: PRJNA626696].

\section{Ethics approval and consent to participate}

Not applicable.

\section{Consent for publication}

Not applicable.

\section{Competing interests}

The authors declare that they have no competing interests.

Received: 23 November 2019 Accepted: 20 May 2020

Published online: 30 June 2020

\section{References}

1. Vega FE. Insect pathology and fungal endophytes. J Invertebr Pathol. 2008; 98(3):277-9.

2. Boomsma JJ, Jensen AB, Meyling NV, Eilenberg J. Evolutionary interaction networks of insect pathogenic fungi. Annu Rev Entomol. 2014;59(1):467-85.

3. Yanhua F, Dov B, Chloe H, Almudena OU, Keyhani NO. Exploiting host molecules to augment mycoinsecticide virulence. Nat Biotechnol. 2012; 30(1):35.

4. Yanhua F, Pereira RM, Engin K, George C, Keyhani NO. Pyrokinin $\beta$ neuropeptide affects necrophoretic behavior in fire ants (S. invicta), and expression of $\beta$-NP in a mycoinsecticide increases its virulence. PLoS One. 2012;7(1):e26924.
5. Li ZZ, Alves SB, Roberts DW, Fan MZ, Delalibera Júnior I, Jian T, et al. Biological control of insects in Brazil and China: history, current programs and reasons for their successes using entomopathogenic fungi. Biocontrol Sci Tech. 2010;20(2):117-36.

6. Uma Devi K, Padmavathi J, Uma Maheswara Rao C, AAP K, MCJBS M. A study of host specificity in the entomopathogenic fungus Beauveria bassiana (Hypocreales, Clavicipitaceae). Biocontrol Sci Tech. 2008;18(10):97589.

7. Ortiz-Urquiza A, Keyhani N. Molecular genetics of Beauveria bassiana infection of insects. Adv Genet. 2016;94:165-249.

8. Wraight SP, Ramos ME, Avery PB, Jaronski ST, Vandenberg JD. Comparative virulence of Beauveria bassiana isolates against lepidopteran pests of vegetable crops. J Invertebr Pathol. 2010;103(3):186-99.

9. Song T-T, Feng M-G. In vivo passages of heterologous Beauveria bassiana isolates improve conidial surface properties and pathogenicity to Nilaparvata lugens (Homoptera: Delphacidae). J Invertebr Pathol. 2011; 106(2):211-6.

10. Wilkening S, Tekkedil MM, Lin G, Fritsch ES, Wei W, Gagneur J, et al. Genotyping 1000 yeast strains by next-generation sequencing. BMC Genomics. 2013;14(1):90.

11. Mburu DM, Maniania NK, Hassanali A. Comparison of volatile blends and nucleotide sequences of two Beauveria bassiana isolates of different virulence and repellency towards the termite Macrotermes michealseni. J Chem Ecol. 2013;39(1):101-8.

12. Valero-Jiménez CA, Faino L, Veld DSIT, Smit S, Zwaan BJ, Kan JALV. Comparative genomics of Beauveria bassiana: uncovering signatures of virulence against mosquitoes. BMC Genomics. 2016;17(1):986.

13. Laurent B, Moinard M, Spataro C, Ponts N, Barreau C, Foulongne-Oriol M. Landscape of genomic diversity and host adaptation in fusarium graminearum. BMC Genomics. 2017;18(1):203.

14. Atwell S, Corwin JA, Soltis NE, Subedy A, Denby KJ, Kliebenstein DJ. Whole genome resequencing of Botrytis cinerea isolates identifies high levels of standing diversity. Front Microbiol. 2015;6:966.

15. Wang S, Leclerque A, Pava-Ripoll M, Fang W. Comparative genomics using microarrays reveals divergence and loss of virulence-associated genes in host-specific strains of the insect pathogen Metarhizium anisopliae. Eukaryot Cell. 2009;8(6):888-98.

16. Xiao G, Ying SH, Zheng P, Wang ZL, Zhang S, Xie XQ, et al. Genomic perspectives on the evolution of fungal entomopathogenicity in Beauveria bassiana. Sci Rep. 2012;2(483):483.

17. Helyar SJ, Hemmer-Hansen J, Bekkevold D, Taylor M, Ogden R, Limborg M, et al. Application of SNPs for population genetics of nonmodel organisms: new opportunities and challenges. Mol Ecol Resour. 2011;11:123-36.

18. Valero-Jiménez CA, Faino L, Spring D, Smit S, Zwaan BJ, van Kan JA. Comparative genomics of Beauveria bassiana: uncovering signatures of virulence against mosquitoes. BMC Genomics. 2016;17(1):986.

19. Weischenfeldt J, Symmons O, Spitz F, Korbel JO. Phenotypic impact of genomic structural variation: insights from and for human disease. Nat Rev Genet. 2013;14(2):125.

20. Grigoriev IV, Martinez DA, Salamov AA. Fungal genomic annotation. Appl Mycol Biotechnol. 2006;6(06):123-42.

21. O'Hanlon KA, Cairns T, Stack D, Schrettl M, Bignell EM, Kavanagh K, et al. Targeted disruption of nonribosomal peptide synthetase pes3 augments the virulence of aspergillus fumigatus. Infect Immun. 2011;79(10):3978-92.

22. Arrebola E, Cazorla FM, Romero D, Pérez-García A, de Vicente A. A nonribosomal peptide synthetase gene (mgoA) of pseudomonas syringae pv. Syringae is involved in mangotoxin biosynthesis and is required for full virulence. Mol Plant-Microbe Interact. 2007;20(5):500-9.

23. Cramer RA, Gamcsik MP, Brooking RM, Najvar LK, Kirkpatrick WR, Patterson TF, et al. Disruption of a nonribosomal peptide synthetase in aspergillus fumigatus eliminates gliotoxin production. Eukaryot Cell. 2006;5(6):972-80.

24. Fan Y, Liu X, Keyhani NO, Tang G, Pei Y, Zhang W, et al. Regulatory cascade and biological activity of Beauveria bassiana oosporein that limits bacterial growth after host death. Proc Natl Acad Sci U S A. 2017;114(9):E1578.

25. Ghikas DV, Kouvelis VN, Typas MA. Phylogenetic and biogeographic implications inferred by mitochondrial intergenic region analyses and ITS15.8S-ITS2 of the entomopathogenic fungi Beauveria bassiana and $B$. brongniartii. BMC Microbiol. 2010;10(1):174.

26. Meyling NV, Lübeck M, Buckley EP, Eilenberg J, Rehner SA. Community composition, host range and genetic structure of the fungal 
entomopathogen Beauveria in adjoining agricultural and seminatural habitats. Mol Ecol. 2009;18(6):1282-93.

27. Rehner SA, Posada F, Buckley EP, Infante F, Castillo A, Vega FE. Phylogenetic origins of African and Neotropical Beauveria bassiana s.l. pathogens of the coffee berry borer, Hypothenemus hampei. J Invertebr Pathol. 2006;93(1): $11-21$.

28. Hideo G, Nobuhiko O, Gi KY, Kouya S, Naoko H, Takeshi H, et al. Extracellular secretion of the virulence plasmid-encoded ADP-ribosyltransferase SpvB in salmonella. Microb Pathog. 2003;34(5):227-38.

29. Rosa JLD, Kaufman PD. Chromatin-mediated Candida albicans virulence $\boldsymbol{z}^{2}$. BBA - Gene Regul Mech. 2012;1819(3-4):349-55.

30. Rottner K, Stradal TE, Wehland J. Bacteria-host-cell interactions at the plasma membrane: stories on actin cytoskeleton subversion. Dev Cell. 2005;9(1):3-17.

31. Dionisio G, Kryger P, Steenberg T. Label-free differential proteomics and quantification of exoenzymes from isolates of the entomopathogenic fungus Beauveria bassiana. Insects. 2016;7(4):54.

32. Weiguo F, Bo L, Yuehua X, Kai J, Jincheng M, Yanhua F, et al. Cloning of Beauveria bassiana chitinase gene Bbchit1 and its application to improve fungal strain virulence. Appl Environ Microbiol. 2005;71(1):363-70.

33. Parish T, Smith DA, Kendall S, Casali N, Bancroft GJ, Stoker NG. Deletion of two-component regulatory systems increases the virulence of mycobacterium tuberculosis. Infect Immun. 2003;71(3):1134-40.

34. Miller SI, Kukral AM, Mekalanos JJ. A two-component regulatory system (phoP phoQ) controls salmonella typhimurium virulence. Proc Natl Acad Sci U S A. 1989;86(13):5054-8.

35. Beier D, Gross R. Regulation of bacterial virulence by two-component systems. Curr Opin Microbiol. 2006;9(2):143-52.

36. Gooderham W, Hancock R. Regulation of virulence and antibiotic resistance by two-component regulatory systems in Pseudomonas aeruginosa. FEMS Microbiol Rev. 2010;33(2):279-94.

37. Zahrt TC, Buchmeier N, Maloy SJI. Immunity: effect of mutS and recD mutations on salmonella virulence. Infect Immun. 1999;67(11):6168-72.

38. Voth DE, Howe D, Beare PA, Vogel JP, Unsworth N, Samuel JE, et al. The Coxiella burnetii ankyrin repeat domain-containing protein family is heterogeneous, with C-terminal truncations that influence dot/lcmmediated secretion. J Bacteriol. 2009;191(13):4232-42.

39. Junan L, Anjali M, Ming-Daw T. Ankyrin repeat: a unique motif mediating protein-protein interactions. Biochemistry. 2006;45(51):15168-78.

40. Lamb SA, Rahman MM, McFadden GJV. Recombinant myxoma virus lacking all poxvirus ankyrin-repeat proteins stimulates multiple cellular anti-viral pathways and exhibits a severe decrease in virulence. Virology. 2014;464: 134-45

41. Pullen KE, Ng HL, Sung PY, Good MC, Smith SM, Alber T. An alternate conformation and a third metal in PstP/Ppp, the M. tuberculosis PP2C family Ser/Thr protein phosphatase. Structure. 2004;12(11):1947-54.

42. Wehenkel A, Bellinzoni M, Graña M, Duran R, Villarino A, Fernandez P, et al. Mycobacterial Ser/Thr protein kinases and phosphatases: physiological roles and therapeutic potential. BBA - Proteins Proteomic. 2008;1784(1):193-202.

43. Goodwin SB, Drenth A, Fry WE. Cloning and genetic analyses of two highly polymorphic, moderately repetitive nuclear DNAs from Phytophthora infestans. Curr Genet. 1992;22(2):107-15.

44. Schmieder R, Edwards R. Quality control and preprocessing of metagenomic datasets. Bioinformatics. 2011;27(6):863-4.

45. Li H. Aligning sequence reads, clone sequences and assembly contigs with BWA-MEM, vol. 1303; 2013

46. Li H. A statistical framework for SNP calling, mutation discovery, association mapping and population genetical parameter estimation from sequencing data. Bioinformatics. 2011;27(21):2987-93.

47. McKenna A, Hanna M, Banks E, Sivachenko A, Cibulskis K, Kernytsky A, et al. The genome analysis toolkit: a MapReduce framework for analyzing nextgeneration DNA sequencing data. Genome Res. 2010;20(9):1297-303.

48. Cingolani P, Platts $A$, Wang $L L$, Coon $M$, Nguyen $T$, Wang $L$, et al. A program for annotating and predicting the effects of single nucleotide polymorphisms, SnpEff: SNPs in the genome of Drosophila melanogaster strain w1118; iso-2; iso-3. Fly. 2012;6(2):80-92.

49. Lam-Tung N, Schmidt HA, Arndt VH, MBJMB Q. Evolution: IQ-TREE: a fast and effective stochastic algorithm for estimating maximum-likelihood phylogenies. Mol Biol Evol. 2014;1:1.

50. Purcell S, Neale B, Todd-Brown K, Thomas L, Ferreira MA, Bender D, et al. PLINK: a tool set for whole-genome association and population-based linkage analyses. Am J Hum Genet. 2007;81(3):559-75.
51. Zhang M, Leong HW. Bidirectional best hit $r$-window gene clusters. Bmc Bioinformatics. 2010;11(Suppl 1):1-9.

52. Ana C, Stefan GT, Juan Miguel GG, Javier T, Manuel T, Montserrat R. Blast2GO: a universal tool for annotation, visualization and analysis in functional genomics research. Bioinformatics. 2005;21(18):3674-6.

53. Kanehisa M, Goto S. KEGG: Kyoto Encyclopaedia of genes and genomes. Nucleic Acids Res. 2000;28(1):27-30.

54. Finn RD, Bateman A, Clements J, Coggill P, Eberhardt RY, Eddy SR, et al. Pfam: the protein families database. Nucleic Acids Res. 2013;42(D1):D222-30.

55. Chen K, Wallis JW, Mclellan MD, Larson DE, Kalicki JM, Pohl CS, et al. BreakDancer: an algorithm for high-resolution mapping of genomic structural variation. Nat Methods. 2009;6(9):677-81.

56. Eddy SR. Profile hidden Markov models. Bioinformatics (Oxford, England). 1998;14(9):755-63.

\section{Publisher's Note}

Springer Nature remains neutral with regard to jurisdictional claims in published maps and institutional affiliations.

\section{Ready to submit your research? Choose BMC and benefit from:}

- fast, convenient online submission

- thorough peer review by experienced researchers in your field

- rapid publication on acceptance

- support for research data, including large and complex data types

- gold Open Access which fosters wider collaboration and increased citations

- maximum visibility for your research: over $100 \mathrm{M}$ website views per year

At BMC, research is always in progress.

Learn more biomedcentral.com/submissions 